\title{
Rescuing of Neuroprotective Peptides by Chemical Conjugation with Lipophilic Active Pharmaceutical Ingredients
}

\section{Sara C. Silva-Reis, ${ }^{a^{*}}$ Ivo E. Sampaio-Dias, ${ }^{a}$ Xerardo García-Mera, ${ }^{b}$ José E. Rodríguez-Borges}

a) LAQV/REQUIMTE, Department of Chemistry and Biochemistry, Faculty of Sciences, University of Porto, Porto, Portugal; b) Department of Organic Chemistry, Faculty of Pharmacy,

University of Santiago de Compostela, Santiago de Compostela, Spain.

*sarac.silva-reis@hotmail.com

KEYWORDS: Neuropeptides; Neurodegenerative diseases; Conjugation; Synergistic effect.

\section{ABSTRACT}

Considering the absence of effective pharmaceuticals to cope with the neurodegenerative processes, our aim was the rescue of Glypromate ${ }^{\circledR}$ by the synthesis of conjugates with this neuropeptide using lipophilic active pharmaceutical ingredients (APIs).

\section{INTRODUCTION}

Glypromate $^{\circledR}$ is a short peptide obtained by N-terminal cleavage of oxytocin hormone mediated by acid proteases. This neuropeptide displays neuroprotective activity within the CNS in many in vitro and in vivo animal models of NDs, and therefore is of utmost relevance for the development of neuroprotective drugs. However, recent clinical trials conducted for this peptide by Neuren Pharmaceuticals failed due to inefficient oral absorption.

\section{OUR WORK}

In this work (Scheme 1), we aimed at the rescuing of Glypromate $^{\circledR}$ by the development of a bioinspired strategy to overcome the low absorption profile associated to this peptide. Since Glypromate ${ }^{\circledR}$ is metabolized by carboxypeptidases, the appropriated amino acid residue for conjugation with lipophilic active pharmaceutical ingredients (APIs) is glutamate, which may allow the release of both APIs and the native peptide. Moreover, this strategy may also provide a means to explore neuroprotective synergism and potentiate the permeability across the blood-brain barrier, potentiating their pharmacological action. For this purpose, small bioactive amines were selected as appropriated APIs for conjugation considering their high lipophilicity and relevance in clinic as therapeutics in the treatment of NDs. Additionally, polar exposed groups were masked to maximize the lipophilicity profile of the conjugates (Scheme 2).

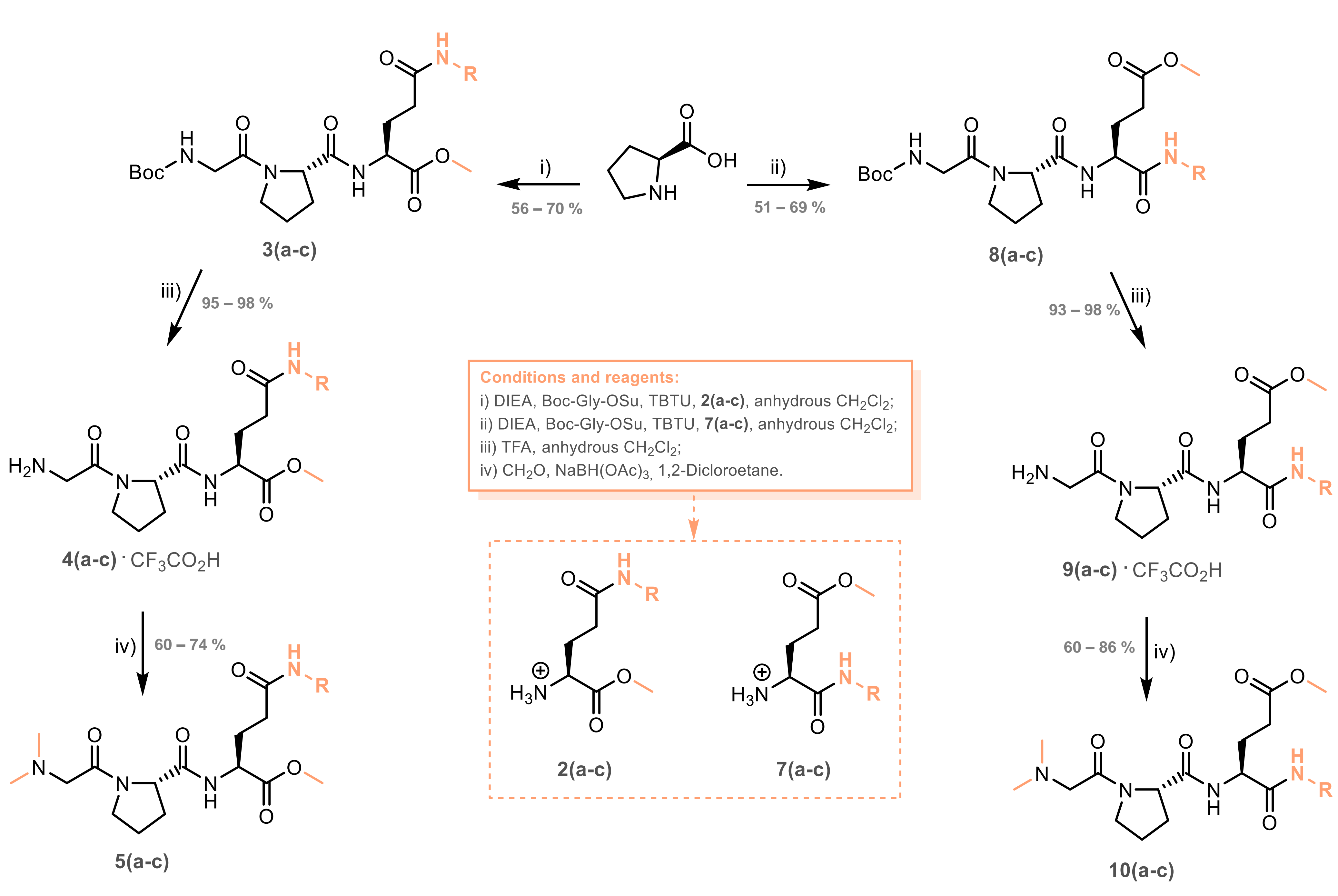

Scheme 2. Synthesis of Glypromate ${ }^{\circledR}$ conjugates.

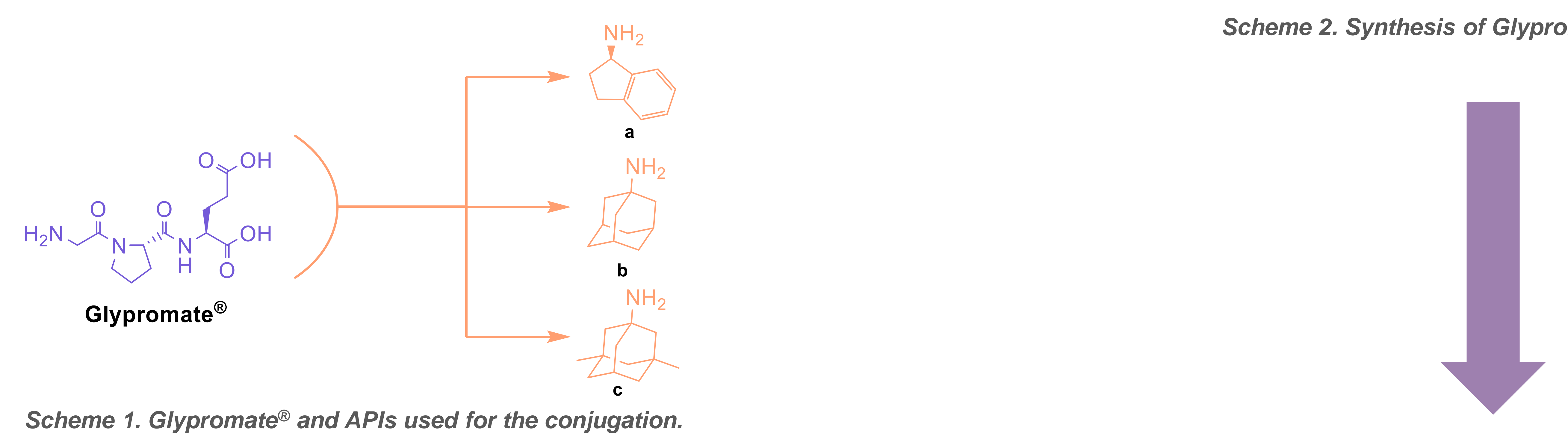

\section{RESULTS}

Preliminary results showed that a conjugate of Glypromate ${ }^{\circledR}$ with aminoindane increased cell viability by $26 \%$ and there was also a synergistic effect since this conjugate demonstrated better neuroprotection compared to its controls. All the conjugates are being biologically evaluated to ascertain their neuroprotective potential using several in vitro models of $A D$ and $P D$. The best conjugates will be then used in vivo models to provide proof-of-concept.
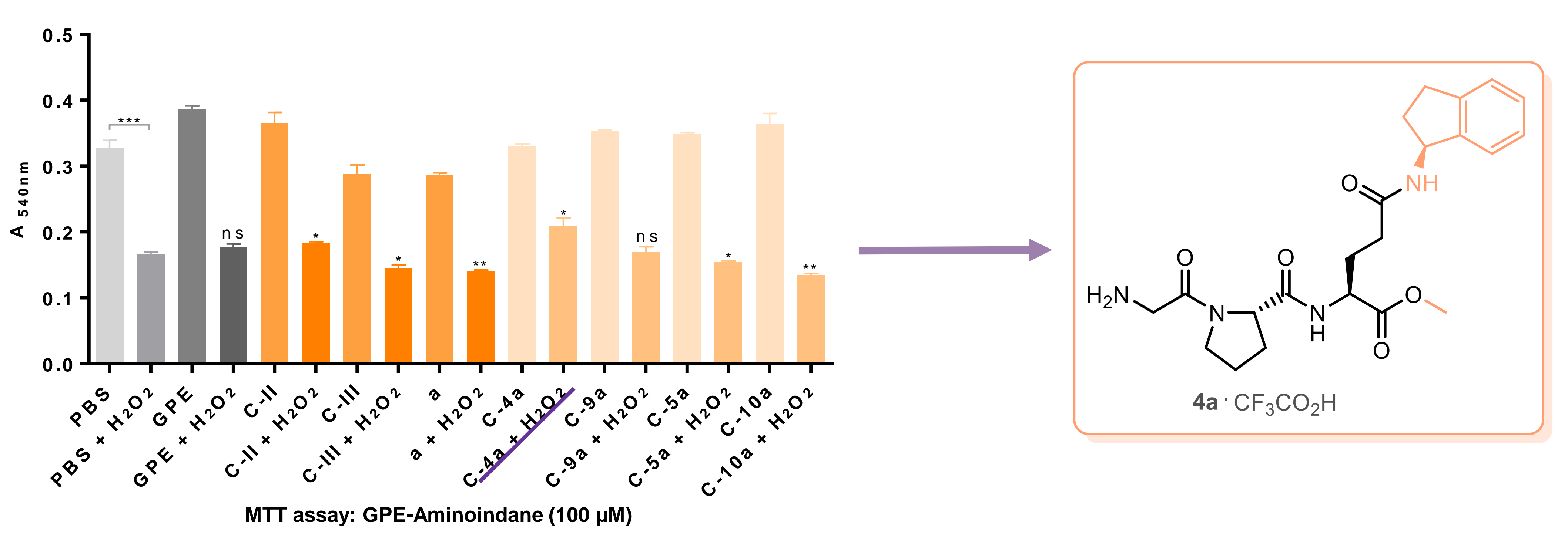

\section{Acknowledgements}

This research was funded by Fundação para a Ciência e Tecnologia (FCT, Portugal), through grants UIDB/50006/2020 (to LAQV-REQUIMTE Research Unit) and project grant PTDC/BIAMIB/29059/2017. Sara C. Silva-Reis is thankful to the FCT for the grant SFRH/BD/147463/2019. 